\title{
CONDIÇÕES DE SAÚDE DO CUIDADOR FAMILIAR DO IDOSO DEPENDENTE
}

\author{
Raimunda Magalhães da Silva ${ }^{1}$, Antonia Rozângela Souza de Oliveira², Luiza Jane Eyre de \\ Souza Vieira ${ }^{1}$, Christina César Praça Brasill e Maria Helena de Agrela Gonçalves Jardim ${ }^{3}$ \\ 1e2Universidade de Fortaleza, Fortaleza, Ceará, Brasil.rmsilva@unifor.br, janeeyre@unifor.br \\ cpraca@unifor.br,Igrozangela@gmail.com \\ 3Universidade da Madeira - Portugal. hjardim@uma.pt
}

\begin{abstract}
Resumo. A vivência do cuidador familiar do idoso dependente leva a importantes reflexões sobre as estratégias para o cuidado da sua saúde. Objetivou-se identificar os aspectos relacionados a condição de saúde do cuidador familiar de idosos dependentes no domicílio e as implicações para o autocuidado. Realizou-se um estudo descritivo, qualitativo, nos domicílios de 10 cuidadores de idosos, em Fortaleza, Ceará, Brasil, de junho a agosto de 2019. As entrevistas foram gravadas, transcritas e organizadas, a partir da análise de conteúdo na modalidade temática. Os 10 cuidadores eram filhos e esposa dos idosos, sendo nove do sexo feminino, com idades de 35 a 72 anos. Dentre os participantes, quatro eram da cidade de Fortaleza e seis de outras cidades do estado do Ceará. As condições de saúde do cuidador apresentam limitações e a protelação do autocuidado é frequente em todos, visto que a dependência do idoso dificulta que o cuidador acompanhe regularmente a sua própria saúde. Ao final, verificou-se que a sobrecarga de trabalho, a idade avançada do cuidador e a falta de apoio físico e afetivo podem ser as principais causas de problemas nas condições de saúde do cuidador.
\end{abstract}

Palavras-chave: Condições de Saúde; Cuidadores; Idoso Dependente; Autocuidado.

\section{HEALTH CONDITIONS OF THE FAMILY CAREGIVER OF THE DEPENDENT ELDERLY}

\begin{abstract}
The experience of the family caregiver of the dependent elderly person leads to important reflections on the strategies for their own health care. The objective was to identify the aspects related to the health conditions of the family caregiver of dependent elderly people at home and the implications for self care. A descriptive, qualitative study was carried out in the homes of 10 elderly's caregivers, in Fortaleza, Ceará, Brazil, from June to August 2019. The interviews were recorded, transcribed and organized, based on content analysis in the thematic modality. The 10 caregivers were sons, daughters and wife of the elderly, nine of whom were female, aged from 35 to 72 years old. Among the participants, four were from Fortaleza and six from other cities in the state of Ceará. The health conditions of the caregiver have limitations and the delay in self care is common in all, since the dependence of the elderly makes it difficult for the caregiver to regularly monitor his(her) own health. It was concluded that the work overload, the caregiver's advanced age and the lack of physical and emotional support can be the main causes of problems in the caregiver's health conditions.
\end{abstract}

Keywords: Health Status; Caregivers; Frail Elderly; Self Care.

\section{INTRODUÇÃO}

O Brasil registra cerca de 29.374 milhões de idosos, correspondendo a $14,3 \%$ da população do país. Muitos desses idosos dependem de cuidados especiais e demandam um número ascendente de cuidadores (Brasil, 2019). É consenso que a transição demográfica e o consequente aumento da expectativa de vida da população ampliam o surgimento de doenças crônicas não transmissíveis, de déficit cognitivo e da incapacidade 
funcional, que favorecem a dependência de cuidados domiciliares, e maior atenção dos familiares com os idosos dependentes (Couto, Castro, \& Caldas, 2018).

Sabe-se que os diversos rearranjos, estruturas e dinâmicas das famílias potencializam o desafio da oferta de um cuidado integral e ininterrupto. Cuidado este, muitas vezes, imerso em tensões intergeracionais, pois cuidar de uma pessoa idosa dependente exige das famílias a aquisição de conhecimentos e condutas no desempenho desta função. Estudiosos asseveram que o cuidador deve receber orientações de como proceder durante os cuidados e o suporte em domicílio, por meio de visitas periódicas de equipe multiprofissional para orientar os procedimentos assistenciais (S. V. C. Areosa, Henz, Lawisch, \& R. C. Areosa, 2014).

O cuidado torna-se mais complexo, na medida em que a incapacidade funcional do idoso progride e a necessidade de um cuidador é real, ficando, na maioria das vezes, ao encargo da família assumir os múltiplos papeis no desempenho desse cuidado. Essa responsabilidade, frequentemente, é realizada por único familiar, tornando a tarefa exaustiva e estressante (Anjos, Boery, \& Pereira, 2014).

O processo de cuidar de um idoso em contexto domiciliar pode impactar o dia a dia do cuidador familiar, levando a dificuldades de ordem emocional, física, econômica e social, com consequente risco à sua saúde e bem-estar (T. S. O. Sampaio, L. S. Sampaio, \& Vilela, 2019).

Entende-se que a relação afetiva que antes era de reciprocidade, passa a ser uma relação de dependência, na qual o cuidador familiar desenvolve atividades relacionadas ao bemestar físico e psicossocial do idoso. Nesse contexto, o cuidador absorve várias atividades, havendo a sobreposição de responsabilidades, que incorrem na restrição de cuidados com a própria vida em várias dimensões (Anjos et al., 2014), o que leva a prejuízos importantes no autocuidado. Compreende-se como autocuidado o ato da pessoa cuidar de si e desenvolver ações de proteção e promoção da saúde, prevenção de riscos e tratamento adequado e precoce (McEwen \& Wills, 2016). Esta definição permite a elaboração de padrões ou metas a serem seguidos em prol da continuidade do bem-estar permanente dos cuidadores de idosos dependentes.

Alinhado a esse desafio inscrito em âmbito global, o presente trabalho discorre sobre a vivência dos cuidadores familiares de idosos dependentes, com ênfase no contexto em que 
estão inseridos, destacando as estratégias utilizadas para o cuidado da própria saúde. Para tanto, questionou-se: como esses cuidadores percebem a sua saúde e praticam o autocuidado.

Acredita-se que este estudo contribui para ampliar o conhecimento sobre o tema, subsidiar novas pesquisas e instigar a elaboração e/ou a reorientação de políticas públicas de apoio social para a oferta do cuidado ao idoso e ao cuidador familiar. Nessa lógica, objetivou-se identificar os aspectos relacionados a condição de saúde do cuidador familiar de idosos dependentes no domicílio e as implicações para o autocuidado.

\section{METODOLOGIA}

Trata-se de um estudo qualitativo, exploratório e descritivo que possibilitou uma maior aproximação com o cotidiano das pessoas, das suas experiências, vivências e expressões subjetivas, considerando as singularidades contextuais (Minayo, 2014). Desse modo, essa abordagem compreende as ações dos participantes como artifícios culturais que exercitam o agir, o refletir e reprogramar, ou transformar o significado que são atribuídos à vida no âmbito social, familiar e de relações (Minayo \& Costa, 2019).

Considerando as ações para a saúde, optou-se por usar os conceitos da teoria do déficit do autocuidado de Orem, especificando prioritariamente o sistema educativo de apoio. Uma das premissas do autocuidado é a crença de que as pessoas se engajam em comunicação e intercâmbios contínuos entre eles mesmos e seus ambientes para permanecerem saudáveis. O autocuidado aponta que a realização de ações pela pessoa ou para ela mesma demonstra o interesse em controlar fatores humanos ou ambientais que afetam o funcionamento e o desenvolvimento pessoal (McEwen \& Wills, 2016).

O processo educativo para o autocuidado poderá ser uma ferramenta para a manutenção da saúde e para o controle de agravos na vida dos cuidadores de pessoas idosas e com a diminuição da capacidade de mobilidade física e cognitiva. Esse sistema possibilita que o cuidador possa realizar ações para prevenção de doenças, identificar possíveis problemas com o seu corpo, e desenvolver mecanismo para promover autonomia do cuidado de si.

O município de Fortaleza/Ceará/Brasil tem sete Secretarias Executivas Regionais (SER I, II, III, IV, V, VI e mais a Regional do Centro/Sercefor). A pesquisa foi realizada na SER VI, área urbana que contém maior abrangência populacional e apresenta número elevado de 
idosos que vivem no domicílio em situação de dependência total do cuidador familiar. Os cuidadores familiares foram selecionados seguindo os critérios de inclusão: idade superior a 18 anos, residente habitual no domicílio, assistência regular ao idoso dependente. Excluíram-se familiares que assistiam o idoso de maneira esporádica, cuidadores contratados e aqueles que estavam ausentes no momento da entrevista.

A coleta de dados ocorreu de junho a agosto de 2019, por meio de entrevista semiestruturada, a qual abordava os seguintes aspectos: variáveis sociodemográficas, história de vida do familiar do idoso; principais problemas vivenciados; relacionamento do cuidador com os outros familiares; dificuldades enfrentado pela família; mecanismos de suporte disponíveis; significados de ter uma pessoa na família com dependência econômica e social; demandas do cuidador familiar aos serviços sociais, de saúde e de direitos; situações de violência na relação com o idoso e com a família; e sugestões do cuidador para que a pessoa idosa tenha o conforto e o tratamento de que precisa.

As entrevistas foram realizadas individualmente, no domicílio, mas em espaço reservado para garantir a liberdade de fala dos entrevistados. Todas as falas foram gravadas com auxílio de um gravador, mediante a autorização prévia do cuidador assim como a assinatura do Termo de Consentimento Livre e Esclarecido (TCLE).

Posteriormente, cada entrevista foi transcrita na íntegra e organizada conforme a análise de conteúdo temática de Bardin (2018) e Minayo (2014). Nesta análise considerou-se as fases da pré-análise, exploração do material, tratamento e interpretação dos resultados. Iniciou-se com a leitura exaustiva a fim de compreender o conjunto de dados e organizálos conforme as convergências e articular com o objeto de estudo. $\mathrm{Na}$ exploração do material fez-se a distribuição das falas que correspondiam aos dados sobre as características dos cuidadores e a protelação para o autocuidado. $\mathrm{Na}$ interpretação buscou-se uma síntese dialogando com o objeto de estudo, objetivo e a literatura a fim de ampliar o conhecimento na temática.

As entrevistas foram encerradas com a repetição das informações coletadas com os dez cuidadores familiares. Os participantes foram identificados pela letra " $C$ " seguida do número da entrevista de (1 a 10) para preservar o anonimato. O estudo foi desenvolvido com base nos princípios legais e éticos adotados nas investigações que envolvem seres humanos, como preconiza a Resolução 466/2012, do Conselho Nacional de Saúde (Brasil, 
2012); tendo sido Foi aprovado pelo Comitê de Ética em pesquisa da Universidade de Fortaleza, sob o parecer n. 1.326.631.

\section{RESULTADOS E DISCUSSÃO}

Apresentam-se resultados que evidenciam a caracterização socioeconômica dos participantes, as condições de saúde do cuidador familiar e as estratégias usadas para a prevenção de riscos e manutenção da saúde, aspectos estes que são permeados pela protelação do autocuidado.

\subsection{Caracterização sociodemográfica dos cuidadores}

A pesquisa envolveu nove cuidadores familiares do sexo feminino e um do sexo masculino, com idades de 37 a 72 anos. Esses dados corroboram a literatura que confere à mulher, prioritariamente, o cuidado familiar, neste caso específico, do idoso dependente, reforçando as assimetrias de gênero legitimadas, cultural e socialmente, que atribuem à mulher a característica de adaptar-se às exigências dos familiares (Cesário, Leal, Marques, \& Claudino, 2017).

Diante da faixa etária dos participantes, evidencia-se que o cuidador familiar pode ser um adulto, uma pessoa de meia idade ou mesmo um idoso, podendo este ser acometido de patologias associadas ou crônicas que, em algumas situações, interferem na oferta de um cuidado qualificado e seguro ao idoso assistido. Essas situações fragilizam o binômio cuidador-idoso, como sinalizam Miranda et al. (2015).

Em relação à naturalidade, observou-se que a maioria dos cuidadores (6) era oriunda de cidades limítrofes do estado do Ceará, enquanto os demais (4), eram naturais do município de Fortaleza, todos residindo também em Fortaleza.

No que se refere ao grau de parentesco com a pessoa cuidada, identificaram-se nove filhos e um cônjuge. Não é incomum, que o filho se torne automaticamente responsável pelos cuidados de seus pais idosos; o que é assumido pelo cônjuge, na impossibilidade de os filhos realizarem essa missão (Linhares \& Viana, 2015). No entanto, essas responsabilidades não são lineares e nem asseguram que sejam isentas de conflitos, ausências, agressões e inquietações, que também fragilizam o cuidado ao idoso dependente e potencializam o nível de insatisfação e desesperança do cuidador (a) (Lacerda, Lacerda, Alves, Lemos, \& Albuquerque, 2019). 
Quanto ao tempo de trabalho como cuidador de idoso, o período oscilou de um a 15 anos. O período de permanência e o tempo dedicado ao cuidado da pessoa idosa dependente influenciam significativamente na qualidade da assistência e gera sobrecarga para o cuidador. Isso pode estar relacionada à escassez de auxílio por parte de outros membros da família, implicando em dedicação e tempo integral do cuidador (Aires et al., 2020).

No que diz respeito ao responsável pelos proventos do idoso, três relataram ser o próprio idoso, cinco eram os filhos, uma a esposa e outro não tinha fonte de renda. Ao assumir a responsabilidade de cuidar do idoso, em algumas situações, automaticamente o cuidador familiar passa também a administrar a parte financeira desta pessoa. Isto, muitas vezes, gera conflitos familiares, devido a desconfiança e a possíveis abusos financeiros realizados pelo cuidador, ou mesmo com gastos relacionados à rotina do cuidado. A violência financeira contra pessoas idosas consiste no uso inapropriado ou ilegal dos seus recursos financeiros ou de seu patrimônio sem a autorização delas, situação comum no âmbito familiar (Henkes \& Areosa, 2019).

\subsection{Saúde e protelação do autocuidado pelo cuidador}

Os dados demonstraram que os cuidadores familiares, por vezes, negligenciam sua saúde em função da saúde do outro. Ainda que busquem atendimento preventivo, com visitas ao médico e a realização de exames, o ciclo não se completa, pois há pendências na busca pelos resultados. Em outras situações, o autocuidado depara-se com barreiras econômicas, com dificuldades na gestão do tempo, na consecução desse olhar para si e fazer valer mudanças no estilo de vida, necessárias à manutenção da saúde em condições crônicas.

[...] fiz uma mamografia para prevenção tem dois meses... Para eu buscar o resultado, eu vou protelando! Para mim sempre vai ficando [para depois]... (C1).

Eu tenho que fazer um check-up do coração, nunca mais eu fiz. [...] veias, varizes, "está" doendo, tem que ir ao médico, eu quero cuidar de mim, mas como vou cuidar, se eu não posso pagar uma pessoa pra ficar com ele [referindo-se ao idoso dependente]? (C6).

Me cuido na medida do possível, eu tenho Diabetes. Mas não faço a caminhada que o médico disse para fazer, mas aí nesse tipo de cuidado eu não tenho como fazer... (C5).

Nas verbalizações dos cuidadores, observa-se o adiamento em relação ao autocuidado. Essas pessoas vão postergando o cuidado por dedicarem a maior parte do seu tempo, ou mesmo das suas vidas, aos seus familiares idosos dependentes. Entende-se que isso 
ocorre devido à capacidade limitada do idoso de desempenhar as atividades básicas da vida diária, exigindo com mais intensidade a atenção e a ação de seus cuidadores.

O cuidador familiar, quando assume a responsabilidade no domicílio pelos cuidados de saúde do seu ente querido, raramente recebe formação ou capacitação para o desempenho desta nova tarefa. Ao lidar com esta realidade, na maioria das vezes, não se sente seguro e preparado para a execução das atividades que envolvem os cuidados no domicílio. Dessa forma, a manifestação de sintomas de sobrecarga passa ser explícita, o que poderá impactar a qualidade do cuidado que irá prestar ao idoso, bem como prejudicar a sua saúde ao longo do tempo (Castro \& Souza, 2016).

Percebeu-se que, em algumas situações, o cuidado é realizado por um único membro da família, sobrecarregando-o e comprometendo as suas condições de saúde física e mental. Nesse contexto, cabe ressaltar que a necessidade de um membro familiar participar do processo de cuidado de um idoso decorre mais de uma imposição circunstancial do que de uma escolha pessoal (Loureiro, Fernandes, Nóbrega, \& Rodrigues, 2014).

Diante dessas situações, verifica-se a necessidade de conhecer a qualidade de vida (QV) dos cuidadores familiares e seus fatores determinantes, para o planejamento de ações de saúde que possam minimizar os efeitos negativos da sobrecarga causada pelo cuidado. Tais ações podem trazer melhorias à qualidade do cuidado dispensado ao idoso e contribuir com a proteção da saúde do cuidador (Rodrigues, Machado, Vieira, Fernandes \& Rebouças, 2014).

Nos depoimentos seguintes, os cuidadores relatam serem portadores de alguma doença crônica e necessitarem de assistência. Além disso, percebe-se a preocupação com relação ao cuidado ofertado aos idosos, em situações que o cuidador familiar precise se ausentar, seja por um processo de saúde ou de outra natureza. Isso gera sentimentos de angústia, aflição e interfere no autocuidado. Os conflitos familiares vivenciados no contexto do cuidado ao idoso também são aspectos referidos pelos participantes, o que torna a rotina diária mais densa, levando, cada vez mais, o cuidador a restringir o cuidar de si.

[...] o cardiologista passou uma tomografia, eletro, exame de tireoide, eu já fiz, são coisas que eu já estou sentindo. Se tiver que enfrentar uma cirurgia, não sei como vai ficar. Isso me perturba às vezes... (C3).

[...] Vou no médico de vez enquanto... [...] o médico disse que eu tinha que fazer alguma atividade física. Estava me sentindo bem melhor, mas, agora, enquadrar nessa rotina de novo eu acho que vai demorar. (C 10) 
A idade dos cuidadores é outra variável relevante, uma vez que, frequentemente, o cuidador pertence à mesma geração das pessoas cuidadas. São idosos com melhores condições de saúde cuidando de idosos dependentes. Nesse cenário, os cuidadores estão suscetíveis aos impactos negativos do cuidado, uma vez que, comumente, apresentam condições de vida semelhante ao idoso cuidado, além de também vivenciarem as mudanças relacionadas ao próprio envelhecimento (Hedler, Faleiros, Santos, \& Almeida, 2016).

Determinadas situações que comprometem a saúde do cuidador podem interferir na qualidade do cuidado prestado ao idoso dependente. Os cuidadores de idosos são chamados de "vítimas ocultas", visto que, em várias situações, além de conviverem com o isolamento, a solidão, a sobrecarga de atividades, as responsabilidades de cuidar e as dificuldades financeiras, ainda lidam com o seu processo de envelhecimento (Anjos et al., 2015).

Percebeu-se que a contribuição de outros membros da família, incluindo os apoios físico, emocional e financeiro por parte de pessoas significativas da família, também se constituiu em um estímulo positivo e relevante no contexto de autocuidado do cuidador (Costa, Castro, \& Acioli, 2015).

É possível identificar no depoimento de C9 (cuidadora nove) que os laços de afeto pela mãe fazem com que se limite a cuidar de si, colocando a sua própria saúde em risco. Como maneira de aliviar o contexto de vida utiliza-se de mecanismos que facilitem o convívio familiar e torne o ambiente tranquilo, minimizando os traumas ocasionados por uma rotina desgastante.

[...] eu quero mais é que minha mãe dure anos e anos, por mais que eu tenha que me debilitar, ficar cada vez mais restrita. Tento não me abalar, eu tento conversar, eu tento manter a paz, porque existe $o$ estresse, existe o cansaço, mas a gente tenta manter. (C9)

Uma cuidadora (C2) refere a adição ao álcool pelo companheiro como sendo um fator que prejudica a sua saúde, levando a glicemia a descompensar nas situações de raiva e estresse. Além de realizar o cuidado ao idoso dependente, existem ainda os conflitos familiares para lidar.

[...] essa bebida do meu marido [referindo-se ao problema]... Porque eu me aborreço, sinto raiva e minha diabete só avança. Porque não me alimento com doces, com gordura, eu procuro fazer o melhor possível. (C2) 
Essas estratégias de enfrentamento ou coping são estratégias utilizadas por pessoas para reduzirem o efeito dos eventos estressantes. Isso pode ocorrer como opção de resolução ou manejo do problema, no intuito de aliviar a tensão provocada pelo estresse no ato de cuidar e controlar as reações emocionais e comportamentais (Marigliano, Silva, Miranda, Rodrigues, \& Gil, 2015).

O adoecimento desses familiares cuidadores pode estar relacionado à intensa convivência com a pessoa idosa, a qual é permeada de situações desgastantes, pois o cuidador tem seu modo de vida desestruturado, visto que apresenta conflitos familiares mais intensos que as pessoas que não exercem essa função (Cesário et al., 2017). Isto mostra a necessidade de programas de suporte formal que devem ser incentivados para promover o equilíbrio familiar, favorecer a redução de conflitos e fornecer aos cuidadores familiares uma referência profissional para orientá-los, capacitá-los e favorecê-los na conciliação do autocuidado com os cuidados ao familiar dependente (Costa et al., 2015).

É possível observar que os aspectos psicológicos dos cuidadores estão afetados, no que se referem à autoestima. Quando questionadas se os mesmos têm tempo para sair, se cuidar ou praticar algo que as faça se sentir bem, estas demonstram tristeza ao tocar no assunto, trazendo em seus relatos palavras de baixa autoestima como podemos constatar nas falas anteriores.

[...] eu nem doente eu posso ficar, eu estou praticamente esquecido, comigo mesmo, porque não tenho tempo nem de ficar doente e sair daqui. (C7)

Não está dando tempo. [...] Acho que está baixa a autoestima... [...] o sorriso, a alegria. Eu tenho a impressão de que posso até estar entrando em um quadro depressivo. (C8)

[...] eu sou muito chorona, se eu for falar de filho, de família, se tiver alguma coisa aí, filho se estiver passando por alguma coisa, aí quando eu vou comentar, já começo a chorar... (C4)

Nota-se que o cuidador sofre uma elevada tensão na sua função, devido a constante alternância de sentimentos, como: tristeza, solidão, falta de apoio tecnológico, emocional, físico, social e, algumas vezes, incapacidade de cumprir as suas obrigações e falta de preparo em assistir ao idoso (Marigliano et al., 2015).

Ressalta-se a relevância dos cuidadores receberem apoio psicológico para aliviar o estado emocional. $\mathrm{O}$ ato de cuidar de idosos acometidos por doenças crônicas pode ocasionar sobrecarga, bem como situações de estresse que poderão causar transtornos para o cuidador, o idoso e seus familiares (Anjos et al., 2015). 
O impacto das situações desgastantes sobre a vida dos cuidadores poderia ser minimizado mediante uma melhor distribuição de tarefas entre os familiares do idoso dependente. Dessa forma, evitar-se-ia a concentração de responsabilidades para um único familiar. A literatura mostra que a orientação e o apoio de uma equipe profissional no redimensionamento do tempo do cuidado e na assistência pode também promover ao cuidador disponibilidade para realizar o autocuidado (Cesário et al., 2017).

Observa-se que os cuidadores não conseguem executar com qualidade os requisitos universais de autocuidado por deixarem essas atividades em segundo lugar em suas vidas, tendo como prioridade o cuidado do outro. Essas modificações no cotidiano do cuidador ocasionam influências para a sua saúde, pois as rotinas não são mais as mesmas quando se tem alguém que precisa do seu cuidado (Nicolato, Santos, \& Castro, 2017).

Entende-se como autocuidado a função humana reguladora que as pessoas realizam deliberadamente por si próprias ou que alguém a execute por eles para preservar a vida, a saúde, o desenvolvimento e o bem-estar. Assim, é fundamental que exista equilíbrio entre o excesso e a carência de cuidado para que a pessoa seja capaz de se autocuidar (Queiróz, Vidinha, \& Almeida Filho, 2014).

Portanto, devido a demanda dos cuidadores em executarem atividades diárias de cuidado ao idoso dependente, estes devem ser incluídos no sistema de apoio-educação. Este sistema permite ao cuidador desenvolver, juntamente com o enfermeiro e/ou outros profissionais da saúde, um plano de cuidados integral e individualizado, para que possam adquirir habilidades e conhecimentos que os auxiliem a realizar o próprio autocuidado, ao mesmo tempo em que cuidam de um membro familiar dependente (Nicolato et al., 2017).

Melhorar as condições de vida e saúde do cuidador, nessa perspectiva, implica dotá-lo de conhecimentos que para sejam capazes de reconhecer em si próprios as suas capacidades físicas e emocionais, no sentido de terem um maior suporte para lidarem com essa nova realidade. Compreende-se que desta forma o cuidador conseguirá realizar suas atividades com segurança e garantir a sua integridade física e psicológica, além de ofertar um serviço com melhor qualidade o idoso assistido (Castro \& Souza, 2016). 


\section{CONSIDERAÇÕES FINAIS}

Neste estudo, pesquisa qualitativa favoreceu a escuta do cuidador sobre a dependência do idoso e as angústias causadas pela ausência de outras pessoas com quem possa compartilhar as atribuições do dia-a-dia relacionadas ao cuidado do idoso dependente. A interpretação qualitativa facilitou o entendimento da abordagem teórica do autocuidado e sua aplicação no contexto de vida e nas ações direcionadas para a preservação da saúde do cuidador. As percepções dos cuidadores evidenciaram, que a dedicação quase que exclusiva do familiar ao idoso, originam uma sobrecarga de trabalho que levam ao comprometimento das condições de saúde, do autocuidado e restringe o tempo para ações de boas práticas de saúde, podendo prejudicar a saúde física e mental dessas pessoas.

Os resultados ampararam a subjetividade dos sentimentos dos participantes e nos conduziu ao aprofundamento das percepções e visões sobre o cuidado de si. Esses fatores podem subsidiar a criação e a implementação de ações de prevenção dos agravos à saúde e a ressignificação de procedimentos que contribuam com melhorias para a qualidade na vida dos cuidadores familiares de idosos dependentes. Os cuidadores geralmente são pessoas com idade de risco para problemas de saúde e que precisam de assistência para a promoção e a manutenção da saúde.

\section{REFERÊNCIAS}

Aires M., Fuhrmann, A. C., Mocellin, D., Pizzol, F. L. F. D., Sponchiado, L. F., Marchezan, C. R., Bierhals, C. C. B. K., Day, C. B., Santos, N. O., \& Paskulin, L. M. G. (2020). Sobrecarga de cuidadores informais de idosos dependentes na comunidade em municípios de pequeno porte. Revista Gaúcha Enfermagem, 41(1), 1-18.

Anjos, K. F., Boery, R. N. S. O., \& Pereira, R. (2014). Qualidade de vida de cuidadores familiares de idosos dependentes no domicílio. Texto Contexto Enfermagem, 23(3), 600-608.

Anjos, K. F., Boery, R. N. S. O., Pereira, R., Pedreira, L. C., Vilela, A. B. A., Santos, V. C., \& Rosa, D. O. S. (2015). Associação entre apoio social e qualidade de vida de cuidadores familiares de idosos dependentes. Ciência \& Saúde Coletiva, 20(5), 1321-1330.

Areosa, S. V. C., Henz, L. F., Lawisch, D., \& Areosa, R. C. (2014). Cuidar de si e do outro: estudo sobre os cuidadores de idosos. Psicologia, Saúde \& Doenças, 15(2), 482-494.

Bardin, L. (2018). Análise de conteúdo. São Paulo: Edições 70.

Brasil. Ministério da Saúde. (2019). Saúde da pessoa idosa: prevenção e promoção à saúde integral. Recuperado de: https://saude.gov.br/saude-de-a-z/saude-da-pessoa-idosa

Castro, L.M., \& Souza, D. N. (2016). Programa de intervenção psicossocial aos cuidadores informais familiares: o cuidar e o autocuidado. Revista-Journal Interações, 12(42), 151-162. 
Cesário, V. A. C., Leal, M. C. C., Marques, A. P. O., \& Claudino, K. A. (2017). Estresse e qualidade de vida do cuidador familiar de idoso portador da doença de Alzheimer. Saúde debate, 41(112), 171-182.

Costa, S. R. D., Castro, E. A. B., \& Acioli, S. (2015). Apoio de enfermagem ao autocuidado do cuidador familiar. Revista Enfermagem UERJ, 23(2), 197-202.

Couto, A. M., Caldas, C. P., Castro, E. A. B. (2018). Cuidador familiar de idosos e o Cuidado Cultural na assistência de Enfermagem. Revista Brasileira de Enfermagem, 71(3), 959-966.

Hedler, H. C., Faleiros, V. P., Santos, M. J. S., \& Almeida, M. A. A. (2016). Representação social do cuidado e do cuidador familiar do idoso. Revista Katálysis, 19(1), 143-153.

Henkes, R., \& Areosa, S. V. C. (2019). Sentidos e Significados da Vida Institucionalizada na Visão de Idosos. Revista Universo Psi, 1(1), 60-80.

Lacerda, M. G. G., Lacerda, G. M., Alves, D. A., Lemos, I. C. S., \& Albuquerque, G. A. (2019). Aspectos envolvidos na assistência prestada ao idoso dependente: percepções dos cuidadores informais. Revista Saúde e Desenvolvimento, 13(15), 35-49.

Linhares, B. N., \& Vianna, L. G. (2015). Fardo do cuidador familiar de idoso com demência de Alzheimer: consequências em sua vida conjugal. Revista de Medicina e Saúde de Brasília, 4(2), 157-65.

Loureiro, L. S. N., Fernandes, M. G. M., Nóbrega, M. M. L., \& Rodrigues, R. A. P. (2014). Sobrecarga em cuidadores familiares de idosos: associação com características do idoso e demanda de cuidado. Revista. Brasileira de Enfermagem, 67(2), 227-232.

Marigliano, R. X., Silva, J. F., Miranda, M. L. J., Rodrigues, G. M., \& Gil, C. A. (2015). Estratégias de autocuidado usadas por cuidadores de idosos: análise de produção científica. Mudanças - Psicologia da Saúde, 23(2), 37-45.

McEwen, M., \& Wills, E.M. (2016). Bases Teóricas de Enfermagem. Porto Alegre, Artmed.

Minayo, M. C. S. (2014). O desafio do conhecimento: pesquisa qualitativa em saúde. São Paulo: Hucitec.

Minayo, M. C. S., \& Costa, A. P. (2019). Técnicos que fazem uso da palavra, do olhar e da empatia: Pesquisa Qualitativa em Ação. Aveiro-Portugal. Ludomedia.

Miranda, A. C. C., Sérgio, S. R., Fonseca, G. N. S., Coelho, S. M. C., Joyce, S. R., Cardoso, C. L., \& Cassiano, J. G. (2015). Avaliação da presença de cuidador familiar de idosos com déficits cognitivo e funcional residentes em Belo Horizonte-MG. Revista Brasileira de Geriatria e Gerontologia, 18(1), 141-150.

Nicolato, F.V., Santos, C. M., \& Castro, E. A. B. (2017). Autocuidado e vivências do envelhecer de cuidadores familiares de idosos: contribuições para enfermagem gerontológica. Tempus, Actas de saúde coletiva, 11(1), 169-186.

Queiróz, P. J. P., Vidinha, T. S. S., \& Almeida, A. J. Filho. (2014). Autocuidado: o contributo teórico de Orem para a disciplina e profissão de Enfermagem. Revista de Enfermagem Referência, 4(3), 157-164.

Resolução n. 466, de 12 de dezembro de 2012. Dispõe sobre diretrizes e normas regulamentadoras de pesquisas envolvendo seres humanos. Recuperado de: http://conselho. Saude. gov.br/resoluções/2012/Reso466.pdf

Rodrigues, J. E. G., Machado, A. L. G., Vieira, N. F. C., Fernandes, A. F. C., \& Rebouças, C. B. A. (2014). Qualidade de vida e sobrecarga de cuidadores familiares de idosos dependentes. Ciencia y Enfermeria, 20(3), 119-129.

Sampaio, T. S. O., Sampaio, L.S., \& Vilela, A. B. A. (2019). Conteúdos e estrutura representacional sobre família para idosos em corresidência. Ciência \& saúde coletiva, 24(4), 1309-1316. 